\title{
Biochemical Properties of Crude Extracellular Proteases from Chromohalobacter salexigens BKL5 and Micrococcus luteus 11A
}

\author{
Ayu Ashari Achmad ${ }^{1,2}$, M. Saifur Rohman ${ }^{1,}{ }^{*}$, Irfan D. Prijambada $^{1}$ \\ ${ }^{1}$ Laboratory of Agricultural Microbiology, Department of Agricultural Microbiology, \\ Faculty of Agriculture, Universitas Gadjah Mada, Yogyakarta 55281, Indonesia \\ ${ }^{2} \mathrm{PT}$. Bernofarm Pharmaceutical, Indonesia
}

\begin{abstract}
In this work, we have reported an enzymatic activity and biochemical properties of extracellular proteases from Chromohalobacter salexigens BKL5 and Micrococcus luteus 11A. C. salexigens BKL5 and M. luteus 11A were previously isolated from Bledug Kuwu mud volcano and dietary industry wastewater treatment, respectively. Both bacterial strains were able to produce extracellular proteases, when grown on minimal agar medium supplemented with $1 \%$ of skim milk. Proteolytic indexes of $C$. salexigens BKL5 and $M$. luteus $11 \mathrm{~A}$ were $2.5 \pm 0.14$ and $2.9 \pm 0.42$, respectively. Both extracellular proteases exhibited optimum enzymatic activity at $\mathrm{pH} 7$, with specific activity of $C$. salexigens BKL5 was $13.3 \%$ higher than that of $M$. luteus $11 \mathrm{~A}$. Optimum temperature for enzymatic activity of both proteases was $45^{\circ} \mathrm{C}$. Metal cofactor preferences assay showed that extracellular protease from C. salexigens BKL5 preferred $\mathrm{Zn}^{2+}$, meanwhile extracellular protease from $M$. luteus 11A mainly preferred $\mathrm{Ca}^{2+}$ ion. Metal cofactor preferences assay also suggested that crude extracellular protease from C. salexigens BKL5 was categorized as metalloprotease, meanwhile crude extracellular protease of $M$. luteus $11 \mathrm{~A}$ was common neutral protease. The enzymatic stability assay against various salt concentrations showed that crude extracellular protease from $C$. salexigens BKL5 was more stable than that of M. luteus 11A.
\end{abstract}

Keywords: Extracellular protease, Chromohalobacter salexigens BKL5, Micrococcus luteus 11A, neutral protease, metalloprotease

\section{Introduction}

Protease is an enzyme that specifically cleaves the peptide bond of polypeptide. Protease has been known to be present in all living organism from bacteria to human and also virus. Furthermore the genomic material contains $2-4 \%$ of protease encoding genes (Puente et al., 2005). It is not surprisingly that protease is indispensable in every organism, since the proteases have significant role many physiological processes such as those associated with metabolisms, cell signaling, defense response, and cell development (Lopez-Otin and Overall, 2002; Sauer et al., 2004; Tripathi and Sowdhamini, 2008). Beside

\footnotetext{
*Corresponding author:

M. Saifur Rohman

Laboratory of Agricultural Microbiology, Department of Agricultural Microbiology, Faculty of Agriculture, Universitas Gadjah Mada, Yogyakarta, Indonesia

E-mail: saifur@faperta.ugm.ac.id
}

of physiologically important, protease has been widely used in various industrial applications such as foods, pharmacy, detergent, textile, and leather. Recent progress shows that protease has been considered for developing of protease-based drug, which works mostly for drug therapy targeted protease activity (Cudic and Fields, 2009; Drag and Salvesen, 2010).

Generally, proteases are categorized into several groups according to the catalytic mechanism, catalytic environment and also catalytic core residues. Based on the catalytic mechanism, proteases could be categorized into two groups, exo- and endo-proteases (Rao et al., 1998). According to the catalytic environment, proteases are separated into acidic, basic, and neutral proteases. This classification is based on the fact that several proteases show the optimum activity either at acidic, basic, or neutral pH (Rao et al., 1998). Identification on the catalytic residues 
responsible for the catalytic activity, it makes possible to further discriminate the members of proteases (Hartley, 1960). Serine, sulfhydryl, and metalloenzyme are an example of the member of protease according to the type of important catalytic residue. Among them, serine protease occupied almost one third of total proteases. This class was originally distinguished by the presentation of what was called by "catalytic triads", which consist of Ser-Asp-His residues (Hedstrom, 2002; Polgar, 2005; Tripathi and Sowdhamini, 2008). According to MEROPS database, the serine proteases consist of four clans include chymotrypsin, subtilisin, carboxypeptidase $\mathrm{Y}$, and $\mathrm{Clp}$ protease (Rawling et al., 2016).

Previously we have isolated one moderately halophilic and one non-halophilic bacteria which showed the proteolysis activity when grown in the minimal media containing skim milk as a substrate. Partial identification based on the 16SrRNA gene sequences indicated that the halophilic bacterium was close to Chromohalobacter salexigens and therefore we have named as $C$. salexigens BKL5 (Rohman et al., 2012). The non-halophilic was close to Micrococcus luteus and then we have named as M. luteus 11A. In this work we have reported the enzymatic activity of two extracellular proteases produced by moderately halophilic bacterium, $C$. salexigens BKL5 and non-halophilic bacterium, M. luteus 11A. Further biochemical properties analyses of those two proteases were also discussed.

\section{Materials and Methods}

\section{Cell and cultures}

Two bacterial strains used in this experiment was $C$. salexigens BKL5 and $M$. luteus 11A. C. salexigens BKL5 was isolated from Bledug Kuwu mud volcano, which has been characterized as moderate halophilic bacterium, while $M$. luteus $11 \mathrm{~A}$ was isolated from dietary industry wastewater treatment. Cultivation of both bacteria was carried out in minimal medium containing $(\mathrm{g} / \mathrm{l}) 13$ $\mathrm{MgCl}_{2} \cdot 6 \mathrm{H}_{2} \mathrm{O}, 20 \mathrm{MgSO}_{4}, 1.1 \mathrm{CaCl}_{2}, 4 \mathrm{KCl}, 0.2$ $\mathrm{NaHCO}_{3}, 0.5 \mathrm{NaBr}$, and $1 \%$ skim milk.

\section{Crude extracellular protease production}

Extracellular proteases were produced by cultivating of $C$. salexigens BKL5 and $M$. luteus $11 \mathrm{~A}$ in $100 \mathrm{ml}$ minimal media supplemented with $1 \%$ skim milk and incubated at room temperature for 48 hours. Culture supernatants were harvested by centrifugation at $8000 \mathrm{rpm}$ for 10 minutes. Supernatants were collected as crude extract extracellular protease and kept at $4^{\circ} \mathrm{C}$ for further analysis. Total protein was determined by using BCA Protein Assay Kit (Novagen).

\section{Enzymatic activity assay}

A modification of the method of Kunitz was used for enzymatic assay by using skim milk as substrate. The enzymatic activity assay was carried out in $0.5 \mathrm{ml}$ of reaction mixture consisted of $400 \mu 1$ of $1 \%$ skim milk substrate in $50 \mathrm{mM}$ phosphate buffer ( $\mathrm{pH} 7.0)$ and $100 \mu \mathrm{l}$ of culture supernatant $\left(\mathrm{OD}_{280} \sim\right.$ 0.1 ). The enzymatic reaction was started by adding culture supernatant and after incubation the reaction was stopped by adding $500 \mu \mathrm{l}$ of cold $10 \%$ of trichloroacetic acids (TCA) solution. The reaction mixture was incubated on ice for 30 minutes and then centrifuged at 10,000 rpm for 10 minutes. An aliquot was carefully collected and subjected for absorbance measurement at $280 \mathrm{~nm}$ (Kunitz, 1946). One unit was defined as the amount of enzyme that increases the absorption at $\lambda=280 \mathrm{~nm}$ by 0.01 . The specific activity was defined as the enzymatic activity per milligram of protein.

To examine the effect of temperature, $\mathrm{pH}$, divalent metal ions, and salts on the enzymatic activity, the enzymatic activity assay was employed by similar method as mention previously by some modifications according to factor to be examined. To examine the effect of $\mathrm{pH}$, the phosphate buffer was used to measure the enzymatic activity at $\mathrm{pH} 5$ to 7 and Tris buffer at $\mathrm{pH}$ 7-9. Divalent metal ions used in this experiment included $\mathrm{CaCl}_{2}, \mathrm{MgCl}_{2}$, and $\mathrm{ZnCl}_{2}$, with the concentration that was varied as follows: $0.5,1,1.5,10$, and $50 \mathrm{mM}$ (Hirokoshi, 1971). To test the protein stability against salinity, the crude 
extracellular proteases from both bacterial strains were incubated in 0 to 5 molar of $\mathrm{NaCl}$.

\section{Results and Discussion}

Crude extracellular protease production from C. salexigens BKL5 and M. luteus $11 A$

Qualitative assay to test the ability of those bacterial strains to produce extracellular proteases were carried out by examining the index proteolytic of both bacterial strains on minimal media containing $1 \%$ skim milk. The assay showed that C. salexigens BKL5 and M. luteus 11A exhibited similar index proteolytic (IP) with the IP values of $2.5 \pm 0.14$ and $2.9 \pm 0.42$, respectively (Table 1 ). In order to further characterize of extracellular protease from both bacterial strains, both strains were grown in liquid minimal medium supplemented with $1 \%$ of skim milk and incubated at room temperature for 48 hours. The harvesting supernatants were used directly for enzymatic assay. By this method of cultivation, the total proteins obtained from the culture supernatants of $C$. salexigens BKL5 and M. luteus 11A were $141.67 \pm 4.99$ $\mathrm{mg} / \mathrm{ml}$ and $141.06 \pm 3.67 \mathrm{mg} / \mathrm{ml}$, respectively. To ensure that the protease enzymes were present in supernatant, we have checked the culture supernatant for the enzymatic activity by using $1 \%$ of skim milk as substrate. Enzymatic activity assay showed that both culture supernatants exhibited comparable enzymatic activity. Enzymatic activity assay showed that the culture supernatants from $C$. salexigens BKL5 and $M$. luteus 11A exhibited enzymatic activity which were determined to be $37.3 \pm 1.20$ $\mathrm{U} / \mathrm{mg}$ and $32.8 \pm 0.91 \mathrm{U} / \mathrm{mg}$, respectively

Table 1. The index proteolytic (IP) of bacterial strains when grown on minimal media agar supplemented with $1 \%$ of skim milk.

\begin{tabular}{ll}
\hline Bacterial strains & Proteolytic Index ${ }^{*}$ \\
\hline C. salexigens BKL5 & $2.5 \pm 0.14 \mathrm{a}$ \\
M. luteus 11A & $2.9 \pm 0.42 \mathrm{a}$ \\
\hline
\end{tabular}

*) The values followed by the same letter were not statistically significant different at $\alpha=0.05$.
(Figure 1). Both culture supernatants showed enzymatic activity against $1 \%$ of skim milk as substrate, therefore, it might be that $C$. salexigens BKL5 and M. luteus 11A produced the similar proteases. In order to check whether the two proteases were similar, we have further characterized the biochemical properties of both proteases.

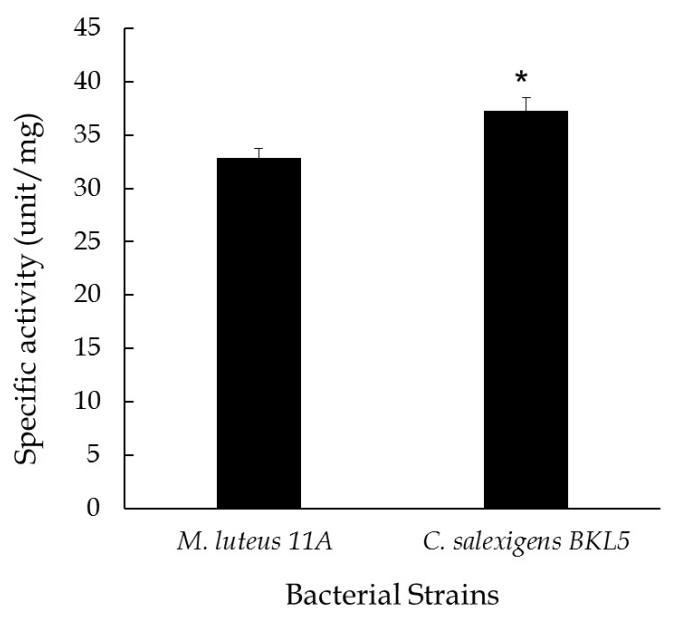

Figure 1. The enzymatic activity of crude extracellular protease from C. salexigens BKL5 and M. luteus 11A grown in liquid minimal media supplemented with $1 \%$ skim milk. Asterisk $\left(^{*}\right)$ indicates statistically significant different at $\mathrm{a}=0.05$.

The effect of temperature, $p H$ and metal cations on enzymatic activity of crude extracellular protease

Effect temperature on enzymatic activity of crude extracellular proteases

Crude extracellular proteases were isolated from the two bacterial strains belong to the mesophilic group. We have induced the proteases production from both bacterial strains at temperature conditions that similar to the optimal growth temperature of both bacterial strains. In order to know the effect of temperature on the enzymatic activity of both crude extracellular proteases, we have examined of both proteases at various different temperature conditions. The result showed that crude extracellular protease from C. salexigens BKL5 exhibited more broad optimum temperature. In contrast, the crude extracellular protease from $M$. luteus 11A exhibited optimum temperature at $45^{\circ} \mathrm{C}$ and when the temperature was increased to $55^{\circ} \mathrm{C}$, 
the enzymatic activity was significantly reduce as much as 55\% from the optimum temperature (Figure 2). The results clearly indicated that crude extracellular protease from C. salexigens BKL5 was more stable compared to that of M. luteus 11A. The result might reflect that both crude extracellular proteases contain several different amino acid residue compositions, which are responsible for protein thermal stability such as proline, arginine, and lysine (Aghajari et al., 2003; Nishio et al., 2003; Prajapati et al., 2007). However, our speculation still needs to be further examined.

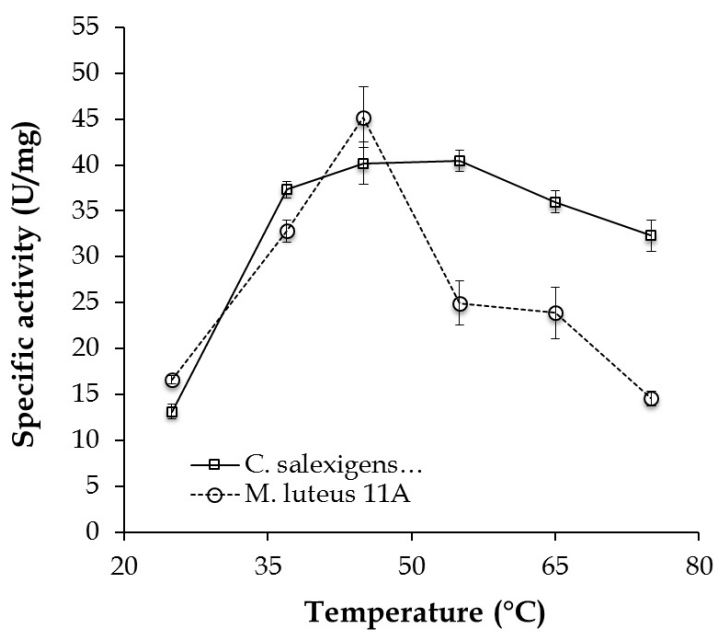

Figure 2. Temperature optimum for enzymatic activity of crude extracellular proteases from $C$. salexigens BKL5 and $M$. luteus 11A. The error bars represent the standard deviations of the corresponding values.

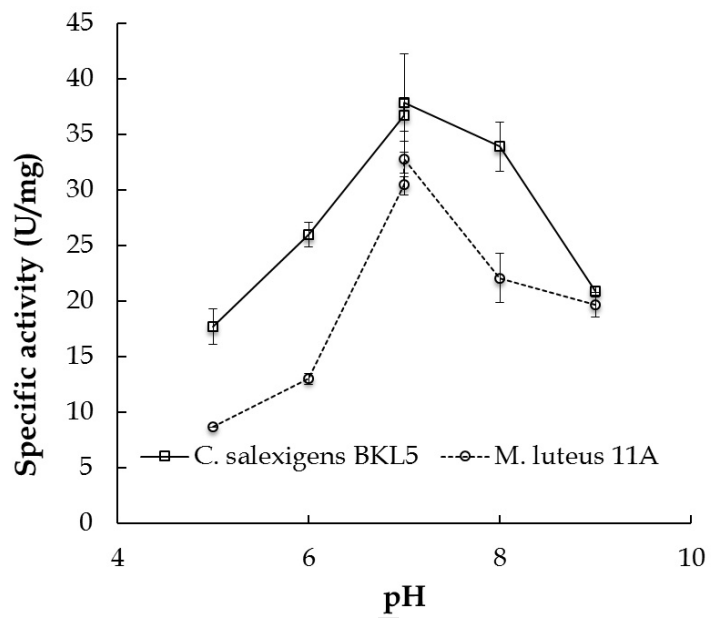

Figure 3. The effect of different $\mathrm{pH}$ of assay conditions on the enzymatic activity of crude extracellular proteases from C. salexigens BKL5 and M. luteus 11A. The enzymatic activity was determined by using the following buffers: phosphate buffer for $\mathrm{pH}$ 5-7 and Tris buffer for $\mathrm{pH}$ 7-9. The error bars represent the standard deviations of the corresponding values.
Effect $p H$ on enzymatic activity of crude extracellular proteases

To test whether the crude extracellular protease from both bacterial strains belong to acid, base, or neutral protease, we have examined the enzymatic activity of both crude extracellular proteases in different $\mathrm{pH}$ conditions. Our result showed that both proteases exhibited optimum enzymatic activity at $\mathrm{pH} 7$ and when the $\mathrm{pH}$ was shifted to more basic then we observed that the enzymatic of both proteases were significantly reduced. Similarly, when we examined at the acidic conditions, both proteases showed less enzymatic activity compared to the basic conditions as well as to the neutral conditions (Figure 3). The results indicated that both proteases belong to neutral protease. Uehara et al., (1974) have reported that the optimum $\mathrm{pH}$ of the neutral protease was 7.3.

Effect of divalent metal ions on the enzymatic activity of crude extracellular proteases

When the enzymatic activity of both crude extracellular proteases were determined in the presence of various concentrations of $\mathrm{CaCl}_{2}, \mathrm{ZnCl}_{2}$ and $\mathrm{MgCl}_{2}$ at $\mathrm{pH} 7$ by using $1 \%$ skim milk as substrate, both proteases exhibited different requirement of the divalent metal ions. Crude extracellular protease from C. salexigens BKL5 preferred $1 \mathrm{mM}$ of $\mathrm{Zn}^{2+}$, while crude extracellular protease from $M$. luteus $11 \mathrm{~A}$ required $10 \mathrm{mM}$ of $\mathrm{Ca}^{2+}$ ion (Figure $4 \mathrm{a}$ and $4 \mathrm{~b}$ ). The result of this study indicated that the crude extracellular protease from $C$. salexigens BKL5 could be categorized as metalloprotease. The common characteristic of metalloproteases was the proteases required Zn as essential metal cofactor (Jiang and Bond, 1992). Furthermore the crystal structure study of psychrophilic metalloprotease of clearly showed that the protease contained $\mathrm{Zn}$, which was located at active site core (Ravaud et al., 2003). Meanwhile crude extracellular protease from $M$. luteus 11A could be categorized as common neutral proteases. As shown in Figure $4 \mathrm{a}$, the crude extracellular protease from C. salexigens BKL5 also exhibited high enzymatic activity when $\mathrm{Ca}^{2+}$ and $\mathrm{Mg}^{2+}$ used as divalent metal cofactor (Figure 4a). Unlike 

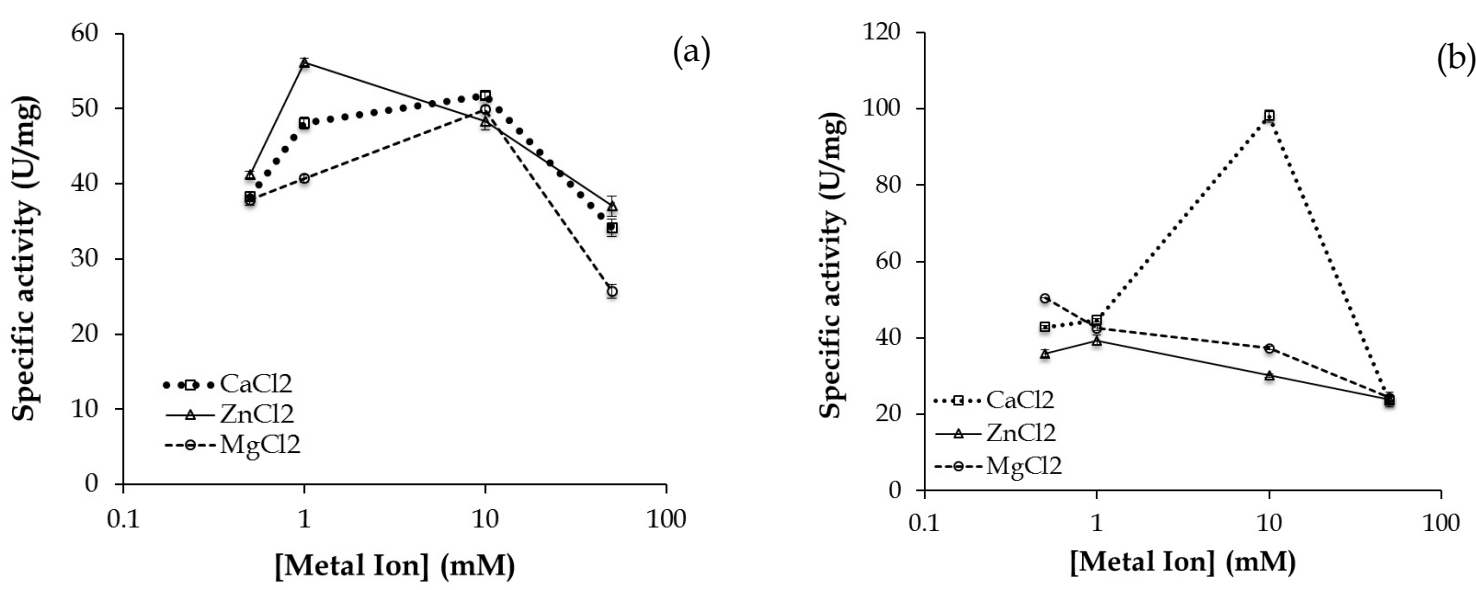

Figure 4. The divalent metal ion preference for enzymatic activity. (a) C. salexigens BKL5 and (b) M. luteus 11A. Divalent metal ion concentration used in this work was $0.5,1,1.5,10$, and $50 \mathrm{mM}$. The error bars represent the standard deviations of the corresponding values.

$\mathrm{Zn}^{2+}, \mathrm{Ca}^{2+}$ was required for protein stability, instead of enzymatic activity (Tajima et al., 1976; Feder et al., 1971).

Furthermore the molar concentration of $\mathrm{Ca}^{2+}$ or $\mathrm{Mg}^{2+}$ was higher than molar concentration of $\mathrm{Zn}^{2+}$. In the case of $\mathrm{Mg}^{2+}$, it might be that the divalent metal ion could replace the role of $\mathrm{Ca}^{2+}$, since both divalent metal ions have similar preference on ligand binding although both divalent metal ions differs ionic radii (Glusker et al., 1999). In the case of thermolysin, a kind of metalloprotease, it stoichiometrically contained 1 g-atom of $\mathrm{Zn}$ and $4 \mathrm{~g}$-atom of Ca per enzyme molecule (Tsuru et al., 1966). Previously, we have reported that the extracellular cellulase

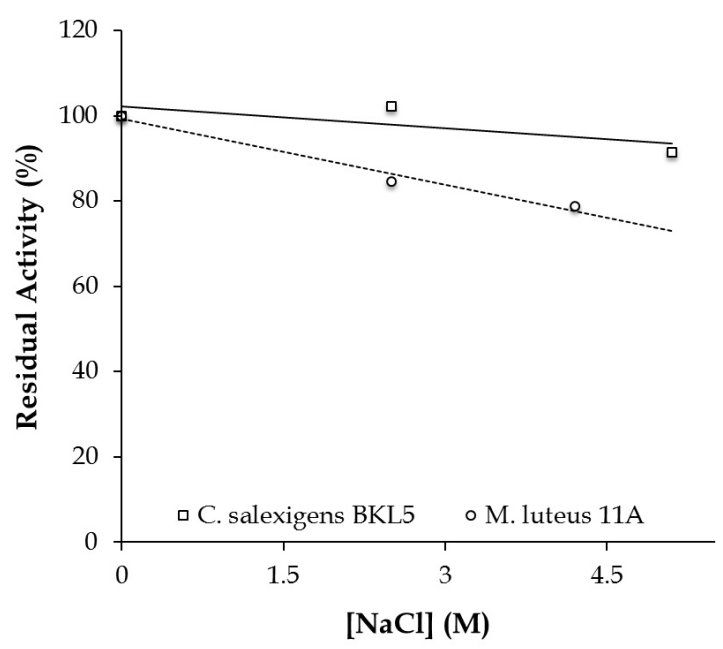

Figure 5. Effect of salt concentration on the enzymatic activity. $\mathrm{NaCl}$ concentrations were varied from 0 to 5 $M$. The error bars represent the standard deviations of the corresponding values. preferred $\mathrm{Mg}^{2+}$ and $\mathrm{Zn}^{2+}$ for enzymatic activity, since both have similar ionic radii (Rohman et al., 2015). It is apparently that the preference of ligand binding formation is more important for structure stability of the proteins, instead for enzymatic reaction. Regarding to the divalent metal ion concentrations, the results clearly indicated that the divalent metal ions required for enzymatic activity of both proteases was 1 to $10 \mathrm{mM}$. Increasing concentration of all the divalent metal ions examined exceeding than $10 \mathrm{mM}$ greatly reduced the enzymatic activity of both proteases. Therefore concentration of divalent metal ions higher than $10 \mathrm{mM}$ was inhibitory for those two crude extracellular proteases (Figure 4).

Effect of salinity on enzymatic activity of crude extracellular proteases

In this work, we have tested the protein stability against the various concentrations of $\mathrm{NaCl}$. The enzymatic activity was determined in the presence of $\mathrm{NaCl}$ with concentration from 0 to $5 \mathrm{M}$ (Figure 5). The result indicated that crude extracellular protease from $C$. salexigens BKL5 showed enzymatic activity that relatively stable with the decreasing rate of enzymatic activity was as much as $1.71 \%$ per molar of $\mathrm{NaCl}$. In contrast, crude extracellular protease from M. luteus 11A exhibited faster decreasing rate of enzymatic activity. The enzymatic activity of crude extracellular protease from $M$. luteus 
11A was decrease by the rate of $5.14 \%$ per molar of $\mathrm{NaCl}$ or 3 times faster than that of C. salexigens BKL5. As expected that the crude extracellular protease from $C$. salexigens BKL5 was more stable than from M. luteus 11A, since $C$. salexigens BKL5 was moderate halophilic bacteria that could grow in the presence of $\mathrm{NaCl}$ up to $20 \%$ (Rohman et al., 2012).

\section{Conclusions}

We have characterized the crude extracellular protease from $C$. salexigens BKL5 and M. luteus 11A. Both proteases were belong to the group neutral protease, however both proteases showed different divalent metal ion requirement. The crude extracellular protease from $C$. salexigens BKL5 preferred $\mathrm{Zn}^{2+}$ as metal cofactor meanwhile crude extracellular protease from M. luteus 11A preferred $\mathrm{Ca}^{2+}$, and therefore, crude extracellular protease from $C$. salexigens BKL5 belongs to the zinc metalloprotease. Crude extracellular protease from M. luteus 11A was less stable than that of $C$. salexigens BKL5 in the presence of high concentration of salt.

\section{Acknowledgements}

This work was partially supported by the grant from the Faculty of Agriculture, Universitas Gadjah Mada, under the program of Faculty of Agriculture Research Grant (Contract No. 1146/PN/TU/2014).

\section{References}

Aghajari, N., Petegem, F. P., Villeret, V., Chessa, J. P., Gerday, C., Haser, R., and Beeumen, J.V. 2003. Crystal structures of a psychrophilic metalloprotease reveal new insights into catalysis by cold-adapted proteases. Prot., 50, 636-647.

Cudic, M. and Fields, G.B. 2009. Extracellular proteases as targets for drug development. Curr. Protein Pept. Sci., 10(4), 297-307.

Drag, M. and Salvesen, G.S. 2010. Emerging principles in protease-based drug discovery. Nat. Rev. Drug Discov., (9), 690-701.
Feder, J, Garrett, L.R., and Wildi, B.S. 1971. Studies on the role of calcium in thermolysin. Biochem., 10(24), 4552-4556.

Hartley, B.S. 1960. Proteolytic enzymes. Annu. Rev. Biochem., 29, 45-72.

Hedstrom, L. 2002. Serine protease mechanism and specificity. Chem. Rev., 102, 4501-4523.

Hirokoshi, K. 1971. Production of alkaline enzymes by alkalophilic microorganism. Part I. Alkaline proteases by Bacillus No. 221. Agric. Biol. Chem., 9, 1407-1414.

Jiang, W. and Bond, J.S. 1992. Families of metalloendopeptidases and their relationships. FEBS Lett., 321(2-3), 110-114.

Kunitz, M. 1946. A spectrophotometric method for the measurement of ribonuclease activity. J. Biol. Chem., 164, 563-568.

Lopez-Otin, C. and Overall, C.M. 2002. Protease degradomics: a new challenge for proteomics. Nat. Rev., 3, 509-519.

Nishio, Y., Nakamura, Y., Kawarabayasi, Y., Usuda, Y., Kimura, E., Sugimoto, S., Matsui, K., Yamagishi, A., Kikuchi, H., Ikeo, K. and Gojobori, K. 2003. Comparative complete genome sequence analysis of the amino acid replacements responsible for the thermostability of Corynebacterium efficiens. Gen. Res., 13, 1572-1579.

Polgár, L. 2005. The catalytic triad of serine peptidases. Cell. Mol. Life Sci., 62, 2161-2172.

Prajapati, R.S., Das, M., Sreeramulu, S., Sirajuddin, M., Srinivasan, S., Krishnamurthy, V., Ranjani, R., Ramakrishnan, C., and Varadarajan, R. 2007. Thermodynamic effects of proline introduction on protein stability. Prot., 66(2), 480-91.

Puente, X.S., Sanchez, L.M., GutierrezFernandez, A., Velasco, G., and LopezOtin, C. 2005. A genomic view of the complexity of mammalian proteolytic systems. Biochem. Soc. Trans., 33, 331-334.

Rao, M.B., Tanksale, A.M., Ghatge, M.S., and Deshpande. V.V. 1998. Molecular and 
Biotechnological Aspects of Microbial Proteases. Microbiol. Mol. Biol. Rev., 62(3), 597-635.

Ravaud, S., Gouet, P., Haser, R. and Aghajari, N. 2003. Probing the role of divalent metal ions in a bacterial psychrophilic metalloprotease: Binding studies of an enzyme in the crystalline state by $\mathrm{X}$ ray crystallography. J. Bacteriol., 185(14), 4195-4203.

Rawlings, N.D., Barrett, A.J. and Finn, R.D. 2016. Twenty years of the MEROPS database of proteolytic enzymes, their substrates and inhibitors. Nucleic Acids Res., 44, D343-D350.

Rohman, M.S., Prijambada, I.D., Indriyani, Y.A., and Hendrosatriyo, H. 2012. Identification of protease producing halophilic bacteria from Bledug Kuwu mud volcano. Indones. J. Biotechnol., 17(1), 35-41.

Rohman, M.S. Pamulatsih, E., Kusnadi, Y., Yuwono, T., and Martani, E. 2015. An active of extracellular cellulose degarding enzyme from termite bacterial endosimbiont. Indones. J. Biotechnol., 20(1), 62-68. Sauer, R.T., Bolon, D.N., Burton, B.M., Burton, R.E., Flynn, J.M., Grant, R.A., Hersch, G.L., Joshi, S.A., Kenniston, J.A., Levchenko, I., Neher, S.B., Oakes, E.S., Siddiqui, S.M., Wah, D.A., and Baker, T.A. 2004. Sculpting the proteome with AAA+ proteases and disassembly Machines. Cell, 119, 9-18.

Tajima, M., Urabe, I., Yutani, K., and Okada, H. 1976. Role of Calcium ions in the thermostability of thermolysin and Bacillus subtilis var. amylosacchariticus neutral Protease. Eur. J. Biochem., 64, 243-247.

Tripathi, L.P and Sowdhamini, R. 2008. Genome-wide survey of prokaryotic serine proteases: Analysis of distribution and domain architectures of five serine protease families in prokaryotes. BMC Genomics, 9(549), 1-28.

Tsuru, D., Kira, H., Yamamoto, T., and Fukumoto, J. 1966. Studies on bacterial protease: Part XIV. Zinc as an essential metal component of neutral protease of Bacillus subtilis var. amylosacchariticus. Agric. Biol. Chem., 80(9), 856-862.

Uehara, H., Yoneda, Y., Yamane, K., and Maruo, B. 1974. Regulation of neutral protease productivity in Bacillus subtilis: Transformation of high protease productivity. J. Bacteriol., 119(1), 82-91. 